Check for updates

Cite this: Mater. Chem. Front., 2020, 4, 1916

Received 14th April 2020

Accepted 19th May 2020

DOI: 10.1039/d0qm00230e

rsc.li/frontiers-materials

\section{Insights into the structural complexity of semi-crystalline polymer semiconductors: electron diffraction contributions}

\begin{abstract}
Martin Brinkmann (D)
Polymer semiconductors are ubiquitous in plastic electronics. Their morphology and structure control electronic and optical properties, hence, the performances of electronic devices such as organic field effect transistors, organic light emitting diodes, organic photovoltaic cells and thermoelectric generators. The molecular complexity of emerging families of polymers such as low bandgap polymers translates into a corresponding structural diversity that needs to be investigated to establish structure-property correlations and ultimately to design more effective materials and devices. Analysis of this structural manifold is challenging and needs appropriate methods of growth and crystallization as well as appropriate structural investigation tools. This contribution is a short review of the recent progress made using electron diffraction to uncover the structural signatures of several representative polymer semiconductors that are widely used in plastic electronics, especially in organic photovoltaic devices.
\end{abstract}

\section{Introduction}

The discovery of conducting, electrically-active polymer materials by Shirakawa, MacDiarmid and Heeger in the late seventies opened a new research area. ${ }^{1}$ The possibility to use polymers for electronic transport is particularly appealing given their unique mechanical properties that make them flexible, lightweight and processable from solution in organic solvents over large surfaces. ${ }^{2}$ Like most materials used in plastic electronics, the processing-structure-property nexus is central and calls for in-depth structural analyses of these polymers in relation to specific processing methods. At the same time, large-scale processing methods such as spin-coating, ink-jet printing or doctor-blading often generate out-of-equilibrium morphologies and ill-defined structures. $^{3}$ Such systems are difficult to analyze in terms of packing and structure due to a limited amount of structural information obtained e.g. via Wide Angle Grazing Incidence $\mathrm{X}$-ray Scattering (GIWAXS). This is why several groups have focused their efforts to control precisely the growth of polymer semiconductors (PSCs) using different strategies that will be reviewed in the following and to analyze the structural features by transmission electron microscopy. ${ }^{4-9}$

Improving device performances has been possible through precision macromolecular synthesis and the design of so-called low-bandgap polymers that associate electron-rich (donor, D) and electron-deficient (acceptor, A) monomeric units in the

Université de Strasbourg, CNRS, ICS UPR 22, F-67000 Strasbourg, France.

E-mail: Martin.brinkmann@ics-cnrs.unistra.fr form of an alternated co-polymer. ${ }^{10}$ The development of low bandgap polymers rests on the possibility to associate a large variety of electron-rich (for instance thiophene, benzodithiophene, indacenodithiophene,...) and electron-poor conjugated blocks (such as diketopyrrolopyrrole, benzothiadiazole,...). This copolymer structure helps adjust light-harvesting, charge transport and transfer properties that are of prime importance in OFETs and solar cells. ${ }^{10,11}$ From a structural point of view, the alternation of $\mathrm{D}$ and $\mathrm{A}$ units in the conjugated backbone provides new and interesting possibilities of interchain interactions. The D and A units can stack in either mixed or segregated manner, which impacts the resulting solid-state electronic properties. In spite of their complex molecular structure, these polymers can form so-called lamellar structures with significant crystallinity. ${ }^{12,13}$ Moreover, side chain engineering is also a method of choice to tune further the polymer's properties. ${ }^{17}$ Another recent strategy to further improve the performances of such PSCs implies fluorination of the polymer backbone that helps to fine-tune the frontier molecular orbital energy levels. ${ }^{14-16}$ This control of the electronic properties is accompanied by sideeffects such as enhanced "aggregation" in organic solvents which is often attributed to the increased propension of polymer backbones to planarize. In the case of n-type polymers, the use of alternating copolymers based on naphthalene diimide (belonging to the family of rylene diimides) has led to air-stable n-type properties in OFETs. ${ }^{12,18}$

Over the last two decades, a large variety of PSCs, including standard homopolymers and alternated D-A low bandgap polymers have been synthesized. However, in-depth structural 

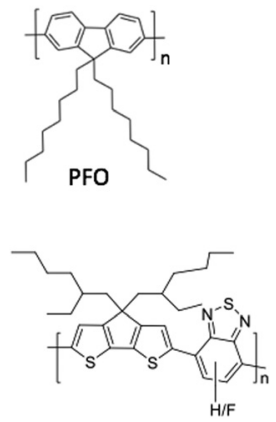

PCPDTBT / F-PCPDTBT

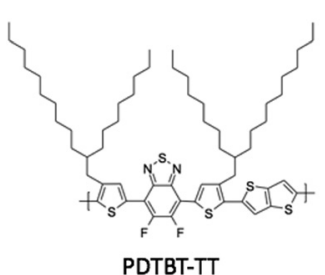

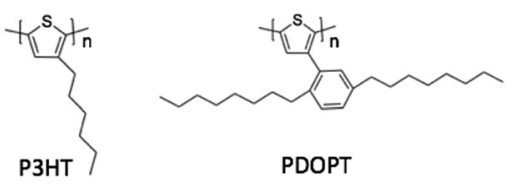

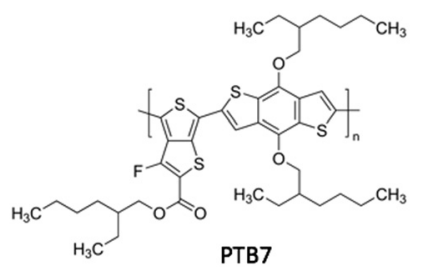

PTB7
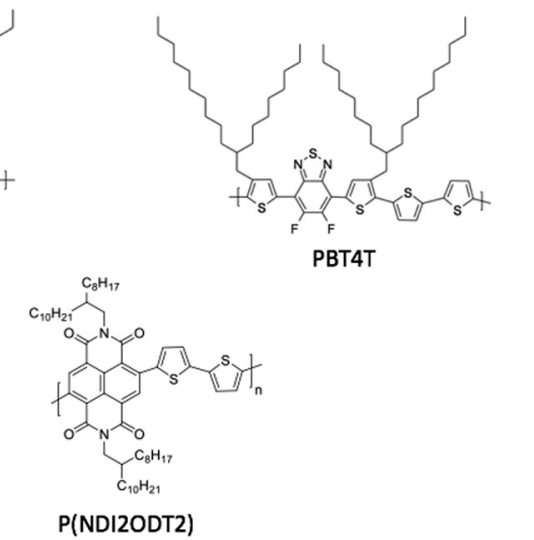

Fig. 1 Semi-crystalline semiconducting polymers the structure of which has been analyzed by electron diffraction. They include homopolymers: poly(9,9'-dioctylfluorene) (PFO), regioregular poly(3-hexylthiophene) (P3HT), poly(3-(2,5-dioctylphenyl)-thiophene) (PDOPT) and alternated copolymers used mainly in organic photovoltaics: poly\{[4,4-bis(2-ethylhexyl)-cyclopenta(2,1-b;3,4- $\left.b^{\prime}\right)$ dithiophene]-2,6-diyl-alt-(2,1,3-benzothiadiazole)-4,7-diyl\} (PCPDTBT and the fluorinated analogue F-PCPDTBT), poly\{4,8-bis[(2ethylhexyl)oxy]benzo[1,2-b:4,5-b]dithiophene-2,6-diyl-alt-3-fluoro-2-[(2ethylhexyl)carbonyl]thieno[3,4-b]thiophene-4,6-diyl\} (PBT7) and poly $\left\{\left[N, N^{\prime}-\right.\right.$ bis(2-octyldodecyl)-naphthalene-1,4,5,8-bis(dicarboximide)-2,6-diyl]-alt-5,5' (2,2' -bithiophene)\} P(NDI2OD-T $)$.

studies could only be performed on a few representative polymers (Fig. 1). A prerequisite to structural investigation is the capability to crystallize such polymers. This contribution aims at giving a synthetic overview on the structures of a few representative polymer semiconductors (Fig. 1) that were determined by a combination of advanced growth control and electron diffraction analysis. The focus will be on the following important issues: (i) representative methods of crystallization and alignment of PSCs, (ii) the structures of several polythiophenes including in particular the forms I and II of P3HT, (iii) typical structural features identified for low bandgap polymers given in Fig. 1 (PCPDTBT, PTB7, PDTBT-TT, PBT4T, P(NDI2ODT2)).

\section{Bringing macromolecules to high order}

The structural analysis of polymer semiconductors implies first of all to develop efficient crystallization methods that yield ordered structures such as single crystals, lamellae, spherulites, fibers or shish-kebab morphologies. These objects can then be analyzed using wide angle X-ray scattering or electron diffraction (ED) in a transmission electron microscope (TEM). Polymers crystallize in a very specific manner, fundamentally different from small molecules, due to the ability of the chains to fold. A polymer chain can re-enter a crystalline lattice after folding and this makes polymer crystallization unique and fascinating. ${ }^{19,20}$ Folded-chain crystallization is common for flexible chains such as polyolefins but is more difficult for rigid or semi-rigid conjugated polymers. However, all PSCs do not exhibit the same chain stiffness that strongly depends on the nature of the aromatic units and of their substituents (fluorine atoms for example) involved in the conjugated backbone. For instance, rigidlike polymers such as poly $\left(9,9^{\prime}\right.$-dioctylfluorene) (PFO) crystallize with extended chains ${ }^{21}$ contrary to the softer chains of poly(3alkylthiophene)s that can fold. ${ }^{22,23}$ Therefore, well-established polymer crystallization methods such as self-seeded growth or epitaxy must be adapted to polymer semiconductors. With their rather stiff $\pi$-conjugated backbones, they can easily aggregate in organic solvents. Different and complementary strategies had to be developed to overcome these difficulties. Fig. 2 depicts the variety of growth methods and the resulting morphologies for different PSCs that have been used to refine polymer structures by electron diffraction.

Reiter and coworkers as well as Ludwigs and coworkers showed that self-seeding approaches can help control the size, shape and number density of crystalline features in thin films of PSCs. ${ }^{4,6}$ The self-seeding approach was introduced originally to overcome the low nucleation probability of polymers. ${ }^{24} \mathrm{~A}$ common way to perform self-seeding consists in using different temperatures for seeding and growth (see Fig. 2II). The seeding temperature $T_{\mathrm{SS}}$ is below the maximum dissolution temperature for which all polymer crystals are dissolved. In a second step, at the crystallization temperature $T_{\mathrm{C}}$ (below $T_{\mathrm{SS}}$ ) the seeds i.e. crystalline fragments that "survived" at $T_{\mathrm{SS}}$ act as seeds for an additional growth. As seen in Fig. 2II, in the case of P3HT, needle-like crystals of form II could be grown from solution in 3-hexylthiophene. The size and surface density of such crystals depends on the temperature of self-seeding $T_{\mathrm{SS}}$ and can accordingly be precisely controlled (see Fig. $2 \mathrm{II}(\mathrm{a}-\mathrm{d}))^{6}{ }^{6}$

Self-seeding can also be performed in the presence of a solvent vapor as an alternative to changing the temperature of seeding and growth. The method implies two steps: (i) a solvent swelling of a thin film at a given vapor pressure close to the dissolution and (ii) a deswelling of the film to promote crystallization. The solvent vapor pressure is controlled by mixing streams of dry nitrogen and of a saturated solvent vapor (see Fig. 2I(a)). Typically, a high solvent vapor pressure $P_{\text {vap }}^{\text {seed }}$ is first used for seeding and then a lower vapor pressure $P_{\text {vap }}^{\text {cryst }}$ for crystallization. Solvent vapor annealing (SVA) proved to be a unique method to prepare controlled morphologies of PSC in the form of spherulites, lamellar crystals and whiskers. ${ }^{4,6}$ SVA allows to grow macroscopic spherulites of PSCs such as P3HT and PCPDTBT with the surface density and size controlled by the vapor pressure $P_{\text {vap }}^{\text {seed } 4}$ The dimensions of these objects are perfectly adapted for a structural analysis by electron diffraction in low-dose mode to preserve the structural integrity 


\section{Solvent Vapor Annealing}

a)
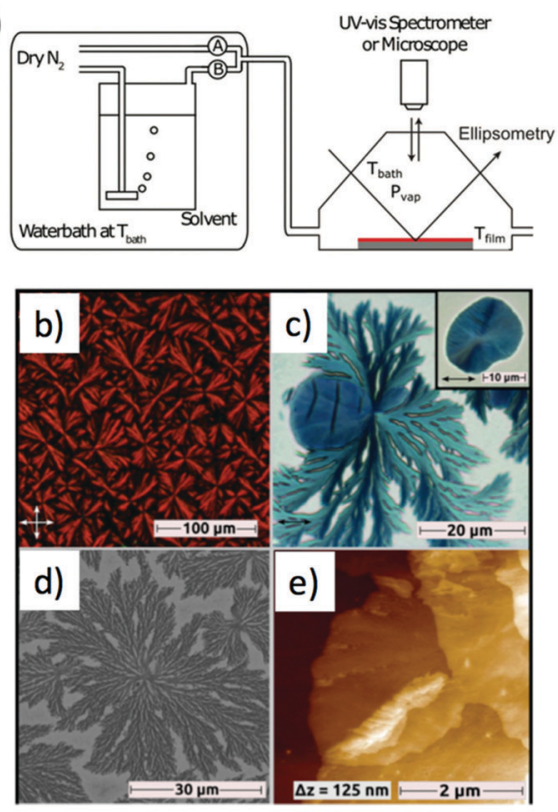

III. Epitaxy

a)
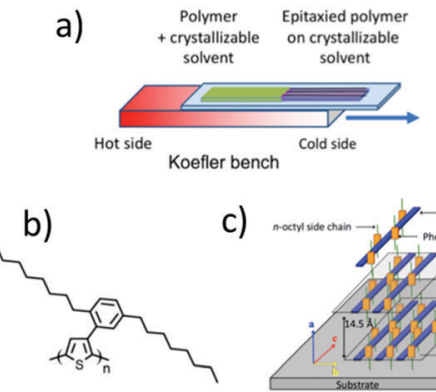

c)
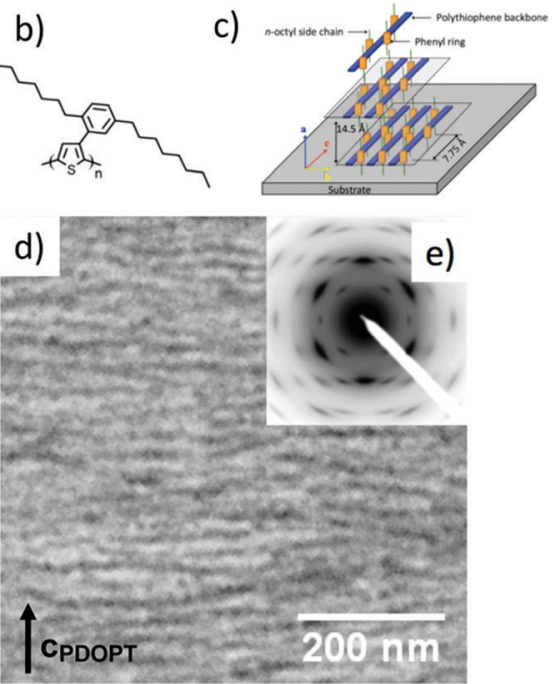

\section{Self-seeded growth}

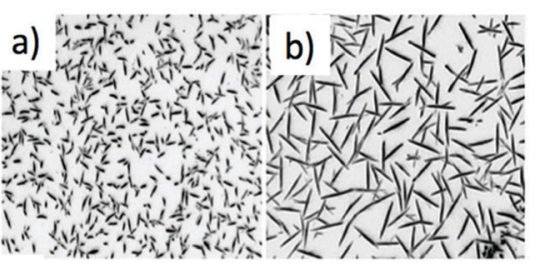

c)
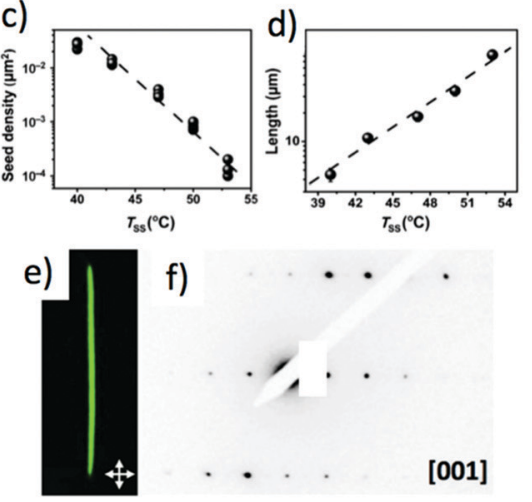

[001]

\section{High temperature Rubbing}

a)

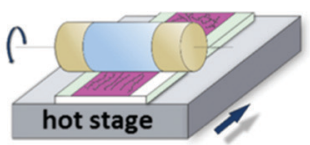

b)
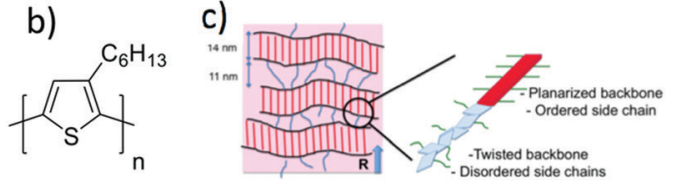

d)

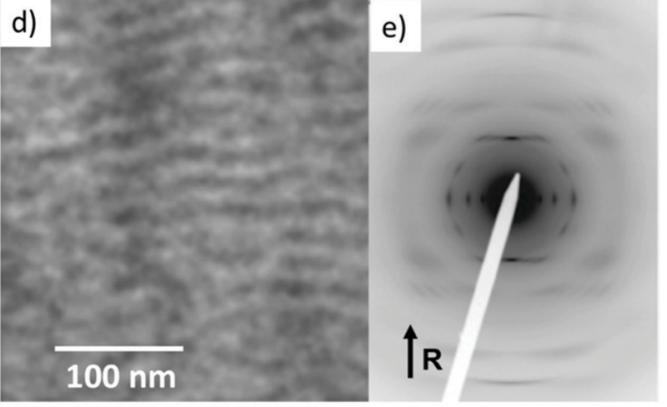

Fig. 2 Comparison of four growth methods for polymer semiconductors: (I) solvent vapor annealing. (a) Schematic illustration of the experimental setup; (b-e) PCPDTBT films after CB-annealing. (b) POM image of a sample with only spherulites (substrate: Si-wafer). (c) POM image of a sample with spherulites and terrace-like structures, inset: magnification of a terrace-like structure. (d) SEM image of the dendritic structure of a spherulite (Au-substrate). (e) AFM height image of a terrace-like structure. (II) Self-seeded growth. (a and b) Optical micrographs (100 $\left.\times 100 \mu \mathrm{m}^{2}\right)$ of P3HT single crystals grown in solutions in 3-hexylthiophene $\left(0.2 \mathrm{mg} \mathrm{ml}^{-1}\right)$ and deposited on a silicon substrate by spin coating. Seeding was performed at $40{ }^{\circ} \mathrm{C}$ in (a), at $43{ }^{\circ} \mathrm{C}$ in (b); growth took place in both cases at $T_{\text {growth }}=32^{\circ} \mathrm{C}$. (c and d) Seed density $N$ and average crystal length versus temperature of seeding ( $T_{\text {ss }}$ ), respectively. (e) Optical micrograph of a P3HT crystal under crossed polarizers and (f) corresponding electron diffraction pattern $\left(T_{\mathrm{SS}}=50{ }^{\circ} \mathrm{C}\right.$ and $T_{\text {growth }}=35^{\circ} \mathrm{C}$ ). (III) Epitaxy. (a) Schematic illustration of the directional epitaxial crystallization method. (b) Molecular structure of PDOPT. (c) Orientation of the PDOPT crystals grown by epitaxy on naphthalene. (d) Bright field TEM image showing the lamellar morphology and (e) corresponding electron diffraction pattern. (IV) High-temperature rubbing. (a) Schematic illustration of the high-T rubbing method. (b) Molecular structure of P3HT. (c) Semicrystalline lamellar structure. (d) Bright field TEM image showing the lamellar morphology. (e) The corresponding electron diffraction pattern ( $R$ is the rubbing direction). Adapted and reproduced with permission from ref. 4, 6, 28 and 37. 
of the objects upon observation in a transmission electron microscope. As seen in Fig. 2I(e), flat-lying lamellar or multi-lamellar crystals with standing polymer chains were grown for PCPDTBT.

A parallel approach is based on the growth of large surfaces of highly oriented and crystallized surfaces of PSCs. Two strategies were used, namely epitaxy and high-temperature rubbing (see Fig. 2III). ${ }^{25-31}$ Epitaxy is defined as a directed growth of a material on an orienting substrate that guides the over-layer's growth along one or several specific crystallographic directions of the substrate. ${ }^{25}$ Contrary to self-seeding that controls the homogeneous nucleation of polymer crystals by adjusting selfseeding temperature or solvent vapor, epitaxy makes use of the nucleating ability of specific substrates. Epitaxy of PSCs is possible on ordered surfaces such as inorganic substrates $(\mathrm{NaCl}$, $\mathrm{KCl}$ ), aromatic molecular crystals (TCB, potassium 4-bromobenzoate $(\mathrm{KBrBz})$, naphthalene) or aligned polymeric substrates (poly(tetrafluoroethylene), poly(ethylene)). ${ }^{7,25-31}$ Epitaxy is determined by a lattice matching between the substrate and the periodicity either along the polymer chain or the distance between chains. ${ }^{26}$ Such a one-dimensional epitaxy is observed for P3HT and PFO grown on 1,3,5-trichlorobenzene (TCB) and for PDOPT on naphthalene (see Fig. 2III). ${ }^{21,26,28}$ In a more marginal and less controlled way, ledges and step edges of certain substrates such as 4-bromobenzoate can also induce nucleation of PSCs such as P3HT. ${ }^{27}$ An original epitaxial crystallization method is directional epitaxial crystallization (see Fig. 2III). The method makes use of a crystallizable solvent in a dual role: (i) in its molten state it is a solvent for the polymer and (ii) in its solid form it is a substrate for epitaxial crystallization (see Fig. 2III(a)). Directional epitaxial crystallization helped establish the semicrystalline morphology of various polymers such as PDOPT and P3HT (periodic lamellar structure seen by TEM in Fig. 2III) and determine their crystal structure. ${ }^{28}$ Advances in directional epitaxial crystallization were obtained by adopting a slow directional growth of the TCB substrate e.g. at a rate of $20 \mu \mathrm{m} \mathrm{s}^{-1}$ leading to extended single-crystal surfaces of the substrate of several $\mathrm{mm}^{2}$.

Not only does epitaxy help in aligning PSCs, it can be used also as an original tool for thin film nano-structuring. When P3HT is grown by epitaxy on TCB in the presence of pyridine, macroscopic aligned fibers of P3HT with a typical "shishkebab" morphology are formed. ${ }^{32}$ The epitaxy of P3HT on potassium 4-bromobenzoate ( $\mathrm{KBrBz}$ ) leads to a unique nanostructuration of the layers. The surface of the aromatic salt $\mathrm{KBrBz}$ undergoes a topographic reconstruction upon annealing of the P3HT film. This results in a regular nanostructured "hill and valley" topography that guides the growth of P3HT domains. ${ }^{27}$

The second alignment strategy is based on strain-orientation of polymer chains that can be enforced in a thin film by stretching, friction-transfer or rubbing. ${ }^{33-37}$ Rubbing is a wellknown method to align polymers such as polyimides and was originally used to fabricate alignment layers for liquid crystal displays. $^{7}$ Of paramount importance to achieve very high orientation of PSCs is the temperature of the polymer film during rubbing. ${ }^{35-37}$ With an adequate rubbing temperature $T_{\mathrm{R}}$ PSC films of remarkable crystallinity and alignment can be formed. ${ }^{36}$ For instance, the crystallinity of P3HT can be tuned from $25 \%$ to $60 \%$ for $T_{\mathrm{R}}$ between $144{ }^{\circ} \mathrm{C}$ and $240{ }^{\circ} \mathrm{C} .{ }^{37}$ The $3 \mathrm{D}$ order parameter that quantifies the degree of in-plane orientation of polymer chains can reach values as high as $0.8-0.9$ for rubbed P3HT films. On a general basis, the rubbing temperature must be in the range between the glass transition temperature and the melting temperature of the polymers. For polymers subjected to a structural transition between a crystalline and a liquid crystalline (LC) phase e.g. PBTTT, rubbing is only possible for temperatures below the transition to the LC phase. ${ }^{35}$ In addition, it has been shown that the optimal rubbing temperature is a function of the polymer molecular weight. As a rule, higher $T_{\mathrm{R}}$ is necessary to align polymers of increasing molecular weight. ${ }^{36}$ This trend has been qualitatively explained by the necessity to disentangle polymer chains of high molecular weight in order to reorganize and crystallize them upon rubbing.

In both cases of epitaxy and high- $T$ rubbing, the main benefit is that the crystalline domains share both a common in-plane direction of the chains and a dominant contact plane of the crystals on the substrate over large surfaces (up to several $\mathrm{mm}^{2}$ ). Accordingly, the diffraction signals of the ordered features can be cumulated in a constructive manner to achieve high intensity (see diffraction pattern in Fig. 2III(e)). Since such oriented films can be prepared over areas much larger than needed for electron diffraction, their investigation is highly facilitated and the inherent issue of beam-damage can be overcome by using nearby structurally equivalent diffracting zones during TEM analysis. This is why, the structure refinement has been possible by electron diffraction following a strategy based on rotation-tilt experiments (see Fig. 3). It allows to collect representative

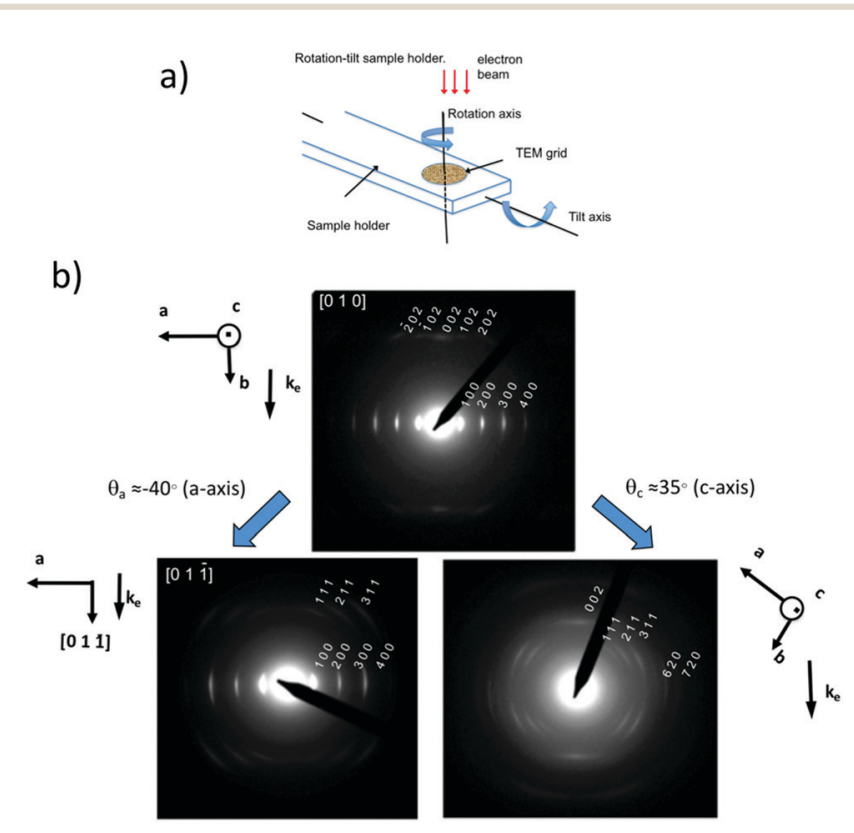

Fig. 3 (a) Schematic illustration of a rotation-tilt TEM sample holder. (b) Rotation-tilt experiment performed on an oriented P3HT film (7.9 kDa) grown by slow-rate directional crystallization in TCB. The sample shows initially a ( 010 ) contact plane ([0 10 ) zone). A tilt around the $a$ axis by $\sim 40^{\circ}$ yields the ED pattern of the [0 $1-1]$ zone with some contribution from the [0 11 1] zone. A tilt of the sample around the $c$ axis by $35^{\circ}$ yields an ED pattern corresponding to the superposition of the $[-1 k 0]$ zones with $k=1,2$ and 3 (adapted from ref. 38). 
diffraction patterns in reciprocal space by bringing the direction of the electron beam along specific zone axes of the crystal or of the oriented thin film (Fig. 3a and b). ${ }^{21,37}$ As an example, in the case of form I of P3HT, typical diffraction patterns for the $\left[\begin{array}{lll}1 & 0 & 0\end{array}\right]$, [ $\left.\begin{array}{lll}0 & 0 & 1\end{array}\right],\left[\begin{array}{lll}0 & 1 & 1\end{array}\right]$ and $\left[\begin{array}{lll}-1 & k & 0\end{array}\right](k=1,2$ and 3$)$ zones were obtained (see Fig. 3). The same approach was followed for poly(9,9'dioctylfluorene) (PFO) grown by directional epitaxial crystallization on TCB. ${ }^{21}$ Collecting a diversity of ED patterns corresponding to different zones is mandatory because it allows to determine the extinction conditions on $(h k l)$ reflections i.e. the selection rules that characterize the space group of a crystal structure. The knowledge of the space group is essential as it defines the symmetry elements that link the monomeric units within a given unit cell. For form I P3HT and PDOPT, this approach helped identify the $P 2_{1} / c$ space group (unique axis $c$ ) whereas for PFO, the $P n b 2_{1}$ space group was determined. $^{21,38}$

Determination of the space group is highly facilitated if the polymer semiconductor films can be obtained with the chain direction oriented both in-plane and normal to the substrate plane. Indeed, in this case, using a TEM it is possible to orient the electron beam parallel or normal to the polymer chains and have access to very important complementary diffraction patterns. Therefore, it is often very useful to combine crystallization and orientation strategies such as solvent vapor annealing that can provide flat-lying lamellar crystals with standing chains and rubbing that produces crystalline structures (lamellae, fibers) with polymer chains lying in the plane of the substrate. ${ }^{4}$

In most cases, structural modeling is necessary to analyze the diffraction data. In the case of P3HT, the refinement strategy is based on a trial-and-error approach that consists in changing a certain number of parameters such as the inclination plane of the alkyl side chain to that of the planarized backbone or the tilting of the backbone to the $\pi$-stacking direction and determining which set of parameters yields the best agreement between experimental and calculated ED pattern..$^{21,38}$ In most cases, this analysis method helped determine tentative structural models that could be ascertained by molecular dynamics and/or energy minimization. ${ }^{28,39,40}$

\section{The structures of polymer semiconductors determined by electron diffraction}

\section{(a) Polythiophenes}

P3HT is the fruit fly among PSCs and has been the center of a considerable research including the investigation of its structure. ${ }^{19}$ The polymorphism of this polymer i.e. its capability to crystallize in various structures, was recognized early on. ${ }^{41,42}$ The polymorphism of P3HT depends on the way the 3-hexyl side chains organize and how this packing influences the stacking of the polythiophene backbones. Most interestingly, P3HT has been the center of numerous structural investigations based essentially on molecular dynamics studies, trying to identify the most stable packing structures. ${ }^{43-46}$ On the down side, the results of such calculated least-energy structures were seldom confronted with experimental structural data on highly oriented and/or crystalline samples. This is mainly due to the intrinsic difficulty to bring "simple" polymer semiconductors such as P3HT to a sufficient level of order to allow for the collection of detailed structural data e.g. by electron diffraction. The progress made in the structural analysis of such polymer is therefore closely linked to the capability of crystallization and ordering of the polymers.

Several studies using electron diffraction helped identify the crystal structures of polythiophenes. Regioregular poly(3hexylthiophene) (P3HT) and poly(3-(2,5-dioctylphenyl)thiophene) (PDOPT) have been the focus of particular interest. ${ }^{28,47,48}$ They are briefly described hereafter.

P3HT. P3HT can crystallize in two polymorphic forms. In form I the side chains are not interdigitated; in form II these side chains are interdigitated. The structure of form I was tentatively refined by electron diffraction on highly oriented films of a low molecular weight fraction aligned by epitaxy on TCB. ${ }^{38}$ The form II of P3HT was refined based on the electron diffraction patterns of single crystals grown by self-seeding (see Fig. 4d-f). ${ }^{6}$ Different structural refinement methodologies were used for both polymorphs. For form I, thin films helped identify the space group by recording diffraction patterns corresponding to different zone axes. Implementing a trial-and-error method yielded a tentative structure for the low molecular weight fraction of form I P3HT. This structure is not induced by the TCB substrate and is identical with that observed in rubbed P3HT films as well as films oriented on potassium 4-bromobenzoate. ${ }^{28,38}$ For form II, the structure was refined by using the sole $\left[\begin{array}{lll}0 & 0 & 1\end{array}\right]$ zone pattern and using the structure of poly(3butylthiophene) proposed by Buono et al. as a starting point ${ }^{49}$ (a rotation-tilt analysis of the ED of the single crystals was impossible for form II due to the high sensitivity of the crystals under the electron beam). Both refined structures confirmed the fact that only form II has interdigitated side chains. The $\pi$-overlaps of the two polymorphs do differ. In form II, short intermolecular distances of $0.33 \mathrm{~nm}$ are found between successive polythiophene chains due to the strong tilt of the polythiophene backbone with respect to the $b$-axis. Most importantly, in form I, the 3-hexyl side chains are oriented in a plane perpendicular to the main chain backbone. The refined model of form I is a so-called "limit-ordered" model that does not correspond to the low-energy or thermally stable structure that would imply some disorder in the packing of alkyl side chains. ${ }^{46}$ In other words, the structural modeling based on ED data yields an ideal and average structure that does not take into account disorder in the arrangement of alkyl side chains. However, such disorder is present in form I as indicated by the presence of very fuzzy and diffuse reflections in the ED pattern of Fig. 2IV for films oriented by high- $T$ rubbing.

PDOPT. Poly(3-(2,5-dioctylphenyl)thiophene) (PDOPT), a phenyl-substituted polythiophene, is highly crystalline with wellordered side chains, in sharp contrast to P3HT form I. ${ }^{28,46-48}$ This $\pi$-conjugated polymer is original because the bulky 2,5dioctylphenyl side groups impede any $\pi$-stacking between the polythiophene backbones. However, despite the absence of $\pi$-stacking, this polymer yields some of the best defined ED patterns of all polythiophenes (see Fig. 5). To analyze its structure, 
Form I. Monoclinic, $\left(\mathrm{P}_{1} / \mathrm{c}\right), \mathrm{a}=1.6 \mathrm{~nm}, \mathrm{~b}=0.78 \mathrm{~nm}, \mathrm{c}=0.78 \mathrm{~nm}$ $\alpha=\beta=90 \mathrm{deg}, \gamma=86.5 \mathrm{deg}$.

a)
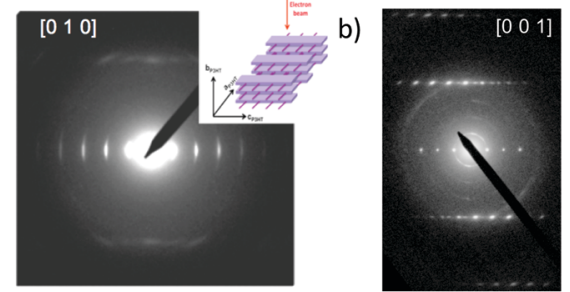

c)

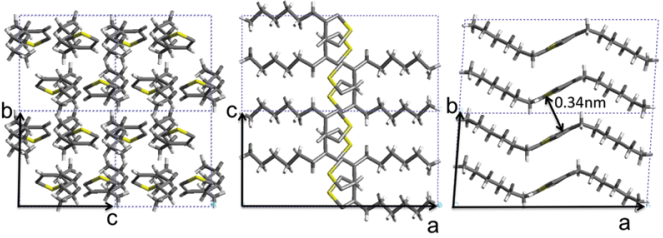

Form II. Monoclinic (P2 $1 / \mathrm{c}) \mathrm{a}=1.29 \mathrm{~nm}, \mathrm{~b}=0.94 \mathrm{~nm}, \mathrm{c}=0.78 \mathrm{~nm}$ $\alpha=\beta=90 \mathrm{deg}, \gamma=68.4 \mathrm{deg}$.

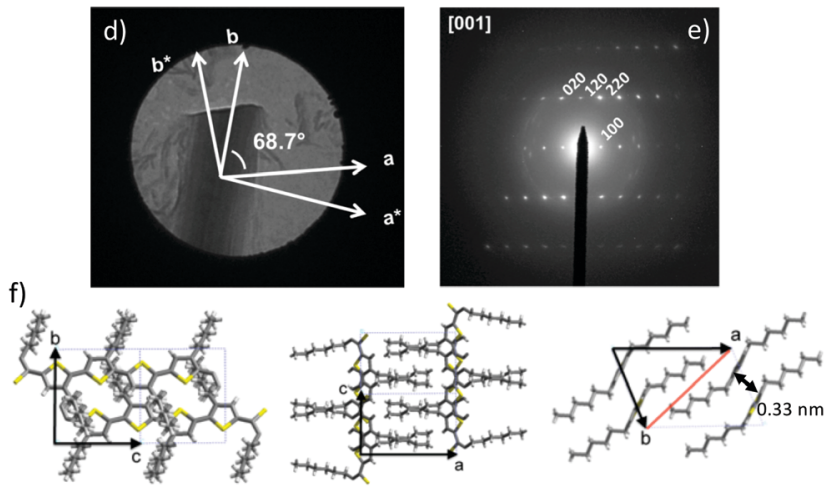

Fig. 4 Comparison of the electron diffraction patterns of P3HT in oriented thin films of form I grown by epitaxy on TCB (a and b) and for a single crystal grown by self-seeding ( $d$ and e). In (c) the crystal structure of form I P3HT is illustrated. In ( $a$ and $b$ ), the orientation of the electron beam with respect to the crystal is highlighted in the insets. In (d), the orientation of the lattice vectors $\mathbf{a}$ and $\mathbf{c}$ is compared to the direction of the vectors in reciprocal space $\mathbf{a}^{*}$ and $\mathbf{c}^{*}$. Reproduced and adapted from ref. 38 (ACS, 2010) and ref. 6 (Wiley-VCH, 2012).

PDOPT, was aligned by directional epitaxial crystallization on naphthalene. The chains orient by 1D epitaxy on the $\left(\begin{array}{lll}0 & 0 & 1\end{array}\right)$ surface of naphthalene with the chain axis direction $c_{\text {PDOPT }}$ parallel to the $a_{\mathrm{Na}}$ axis of naphthalene. This 1D epitaxy leads to the remarkable ED patterns (shown in Fig. 5b) with a wealth of mixed-index reflections implying high order of both polythiophene backbone and side chains. The majority of the film is composed of PDOPT lamellae with polymer chains lying in-plane with a typical $\left[\begin{array}{lll}1 & 0 & 0\end{array}\right]$ zone pattern. In some areas, flat-on lamellae with polymer chains oriented normal to the film plane can be found, that yield a different pattern (from the [001] zone).

Refinement of the crystal structure of PDOPT was accordingly possible. The cell parameters are $a=2.909 \mathrm{~nm}, b=1.045 \mathrm{~nm}$, $c=0.77 \mathrm{~nm}$ and $\alpha=\beta=\gamma=90^{\circ}$ and selection rules identify the space group as $P 2_{1} / c .^{28,50}$ Trial-and-error analysis of the experimental ED patterns yielded a tentative model that was subsequently energy-minimized. ${ }^{28}$ Excellent agreement between calculated and experimental ED was reached (see Fig. 5). The refined structure indicates that the PDOPT backbone is planar despite the absence of $\pi$-stacking and the 2,5-dioctylphenyl side groups are oriented in a plane perpendicular to the polymer chain direction. $\mathrm{N}$-Octyl side chains form a dense and highly ordered sublattice, which explains the multiple mixed index reflections in the ED. A comparison of the sub-lattices of P3HT form I, PBTTT (poly[2,5-bis(3-alkylthiophen-2-yl)thieno[3,2-b]thiophene], another reference thiophenebased polymer) and PDOPT shows that a high ordering of polymer semiconductor chains as a whole is subjected to the ability of the alkyl side chains to form highly ordered sublattices. ${ }^{28}$

\section{(b) The variety of structures formed by alternated donor-acceptor co-polymers}

P(NDI2ODT2). The original structural complexity of alternated copolymers was first studied for the case of rylene diimide copolymers. They consist of an alternation of acceptor units such as naphthalene diimide (NDI) or perylene diimide (PDI) and electronrich donor comonomers such as bithiophene. In particular, poly$\left\{\left[N, N^{\prime}\right.\right.$-bis(2-octyldodecyl)-1,4,5,8-naphthalenedicarboximide-2,6diyl]-alt-5, $5^{\prime}$-(2,2'-bithiophene) $\}$ (PNDI2OD-T2) has been the focus of intense research efforts as it stands as one of the few air-stable n-type polymer semi-conductors (see Fig. 6). ${ }^{13,18,51,52}$

The complexity of ordering in $\mathrm{D}-\mathrm{A}$ alternated copolymers was addressed successfully in the case of P(NDI2OD-T2) by taking advantage of the possibility to align this polymer using different growth methods. Brinkmann and coworkers used both directional epitaxial crystallization in TCB and epitaxy on oriented poly(tetrafluoroethylene) (PTFE) to grow two polymorphs of this polymer, form I and form II (see Fig. 6). ${ }^{53,54}$ Later, a combination of rubbing at $80{ }^{\circ} \mathrm{C}$ and annealing at different temperatures afforded also the two polymorphs of P(NDI2OD-T2) (Fig. 6c and e). ${ }^{54}$ Most importantly, the distinction between the two polymorphs can only be made if the reflections along the chain direction can be observed precisely which implies to work with oriented thin films. The two polymorphs correspond to so-called segregated (form I) and mixed (form II) stacking of the NDI and T2 units (see Fig. 6a). The two forms present two distinct morphologies. The films of form I consist of fibrils whose long axis corresponds to the chain direction whereas the form II films involve a periodic lamellar structure with sharp boundaries between successive lamellae suggesting extended-chain type of crystallization. ${ }^{53-55}$ Form I is characterized by a strong 001 reflection that is typical of the segregated stacking of NDI and T2 blocks. In form II, the $\begin{array}{lll}0 & 0 & 1\end{array}$ reflection is almost absent but a strong 002 reflection is observed. This is the fingerprint of a $c / 2$ shift between successive $\pi$-stacked P(NDI2OD-T2) chains leading to the mixed stacking of NDI and T2. This polymorphism is observed for different types of preparation methods (epitaxy on TCB and PTFE, rubbing, blade coating). It is thus an intrinsic feature of the polymer and is not substrateinduced. Most importantly, the structure could be correlated with the UV-vis absorption and charge transport in OFETs. Polymorphism influences the charge transfer band around $750 \mathrm{~nm}$ of P(NDI2OD-T2) whereas little impact was observed on the electron mobilities in aligned thin films of the two polymorphs. ${ }^{54,55}$ 
a)

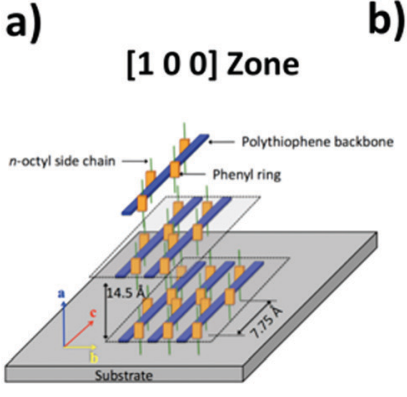

b)

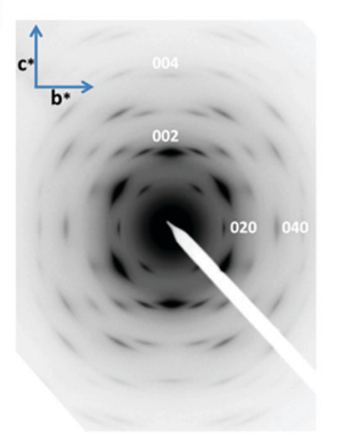

c)

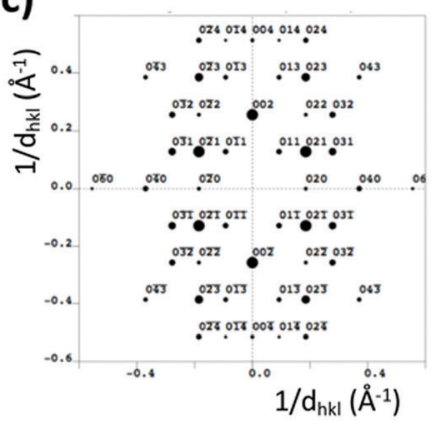

d)

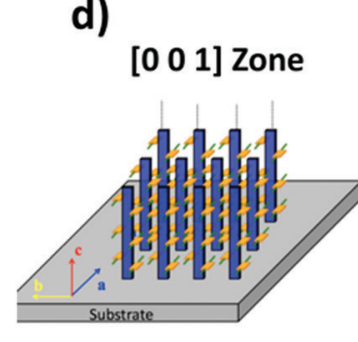

g)
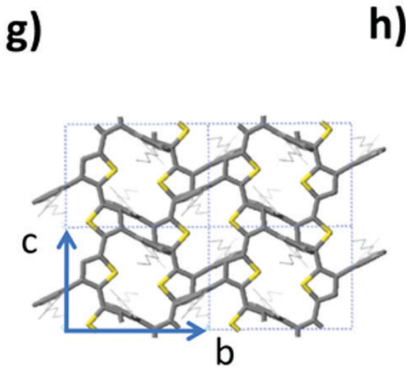

e)

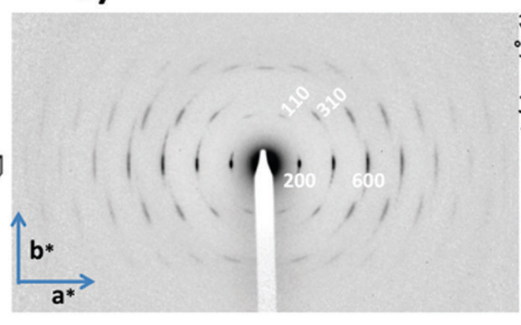

f)

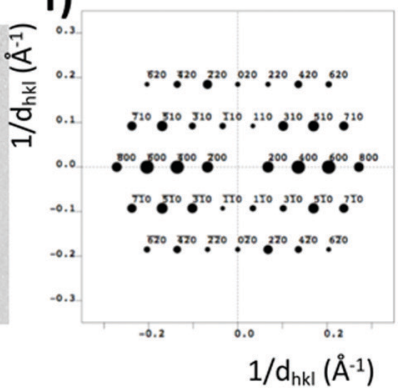

h)

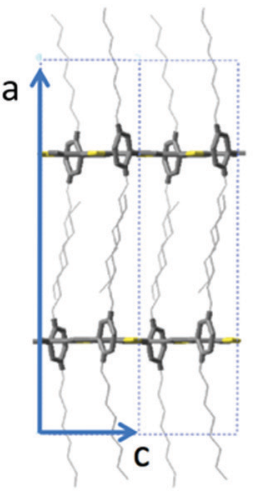

i)

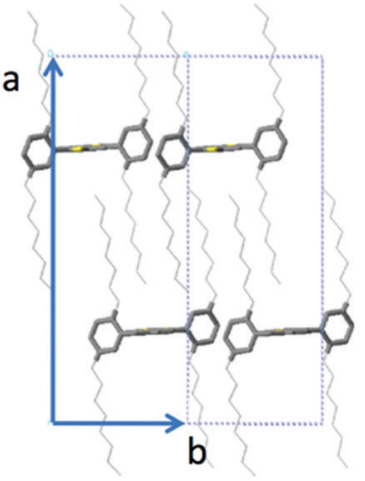

Fig. 5 Comparison of the experimental and calculated ED pattern of highly oriented PDOPT thin films grown by epitaxy on naphthalene. (a-c) For the $\left[\begin{array}{lll}1 & 0 & 0\end{array}\right]$ zone (electron beam parallel to the octyl side chains) and ( $\left.d-f\right)$ for the $\left[\begin{array}{lll}0 & 0 & 1\end{array}\right]$ zone (electron beam along the chain direction). (a) and (d) show schematic illustrations of the crystal orientations with respect to the electron beam (vertical). (g)-(i) are the typical projections of the refined structure of PDOPT along the $a, b$ and $c$ directions of the refined unit cell (reproduced and adapted with permission from ref. 28).

Charge mobility measurements in OFETs using films aligned by rubbing revealed a strong transport anisotropy. The enhanced electron mobilities along the rubbing direction reached values of up to $0.1-0.3 \mathrm{~cm}^{2} \mathrm{~V}^{-1} \mathrm{~s}^{-1} .53$

PBT4T and PDTBT-TT. In the case of P(NDI2OD-T2) the metastable form I composed of segregated stacks of D and A blocks can be transformed into a thermodynamically more stable form II with a mixed stacking of D and A units. Recent progress in the design of PSCs evidenced the beneficial effects of backbone fluorination. Leclerc and coworkers reported that an alternated copolymer made of a fluorinated benzothiadiazole and a 2,5-bis(3-alkylthiophen-2-yl)thieno[3,2-b]thiophene comonomer (DT-TT) has remarkable photovoltaic performances. ${ }^{56} \mathrm{~A}$ structurally closely related polymer, in which the quaterthiophene (4T) unit is used as comonomer, (PBT4T hereafter, also known as PCE11) can also reach PCE of over $10 \% .^{57,58}$ The packing of such alternated copolymers is an important parameter governing their performances in OPV devices. In particular, it is of importance to understand how thermal annealing affects the structure of such polymers comprising a fluorinated benzothiadiazole unit. Moreover, such fluorinated polymers aggregate readily in common organic solvents and hence show characteristic low energy "aggregate-bands", of uncertain structural origin.

In this perspective, Brinkmann and Leclerc studied the polymorphism of these two alternated copolymers, that share a common fluorinated benzothiadiazole unit (BT) as electronwithdrawing group (see Fig. 7). ${ }^{59}$ ED analysis on films aligned by high- $T$ rubbing demonstrated that both polymers crystallize in a segregated stacking mode (Fig. 7a-d). The polymorph is made of segregated stacks of benzothiadiazole and of the comonomers (DT-TT or 4T). However, only the segregated stacks of PDTBT-TT are stable up to a temperature close to the melting. 
a)
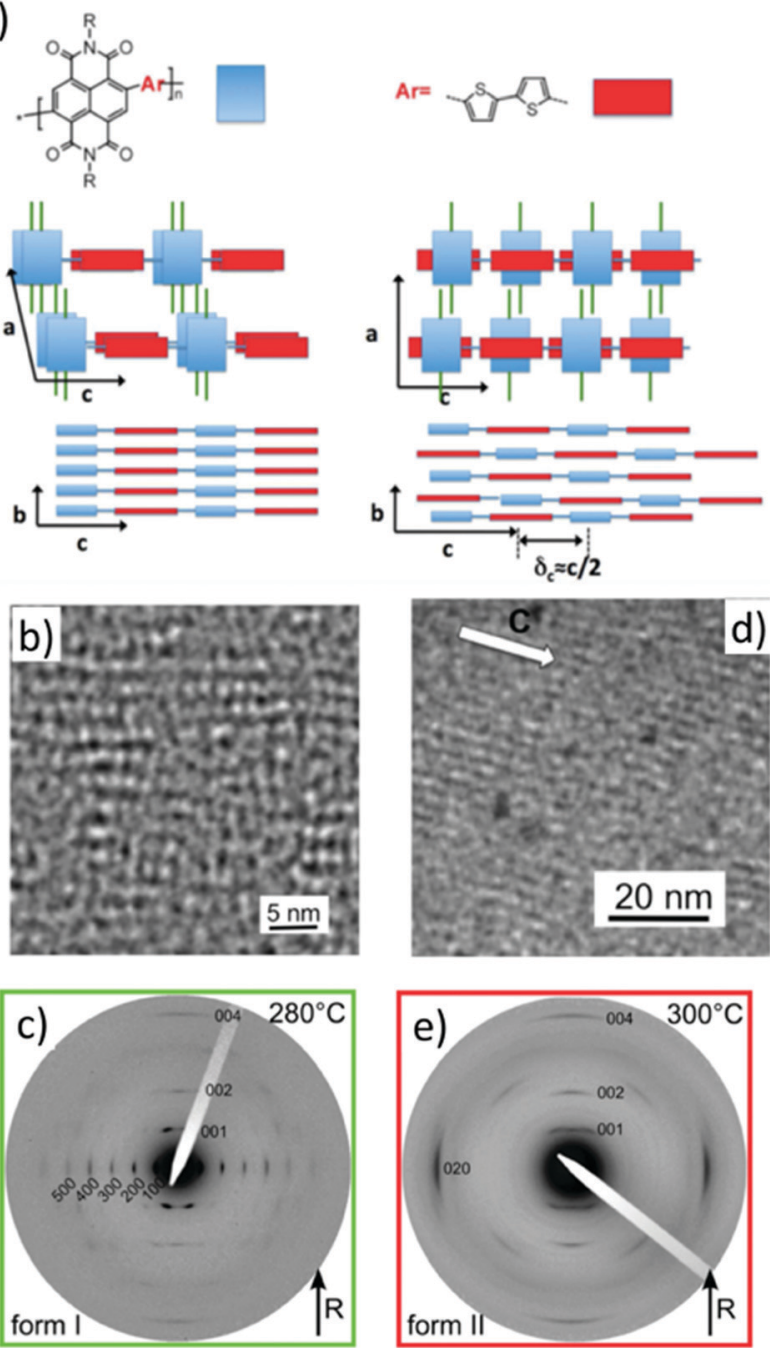

Fig. 6 (a) Schematic illustration of the stacking of NDI and T2 units for form I and II of P(NDI2OD-T2). (b) HR-TEM of oriented thin films of $\mathrm{P}(\mathrm{NDI}$ 2OD-T2) obtained by directional epitaxial crystallization on TCB. (c) Electron diffraction patterns of P(NDI2OD-T2) in form I after one rubbing cycle at $80{ }^{\circ} \mathrm{C}$ and a subsequent thermal annealing at $280{ }^{\circ} \mathrm{C}$. (d) HR-TEM of oriented thin films of P(NDI2OD-T2) in form II obtained by epitaxy on oriented PTFE substrates after annealing at $300{ }^{\circ} \mathrm{C}$ and cooling at $0.5^{\circ} \mathrm{C} \mathrm{min}$ min $^{-1}$ to ambient temperature. (e) Electron diffraction patterns of P(NDI2OD-T2) form II for a film aligned by rubbing at $80{ }^{\circ} \mathrm{C}$ and annealed at $300{ }^{\circ} \mathrm{C}$. Adapted and reproduced with permission from ACS and Wiley and Sons. ${ }^{53,54}$

In the case of PBT4T, the segregated stacking becomes progressively disordered upon thermal annealing at $T_{\mathrm{A}} \geq 250{ }^{\circ} \mathrm{C}$ : the BT units $\pi$-stack in a random manner with the $4 \mathrm{~T}$ co-monomer (see Fig. $7 \mathrm{~d}$ ). It was demonstrated that the so-called low energy "aggregate-band" signals the polymorph with a segregated stacking. This band is highly sensitive to disorder that occurs upon annealing at high temperatures within the stacks of polymer chains. The statistical mixing of $\pi$-overlaps between the BT and 4T units in PBT4T results in a loss of the aggregate band. The comparative study of PBT4T and PDTBT-TT shows that the stability of the segregated stacking depends on the nature of the comonomer and is more favorable for BT-TT (see Fig. 7d). a)

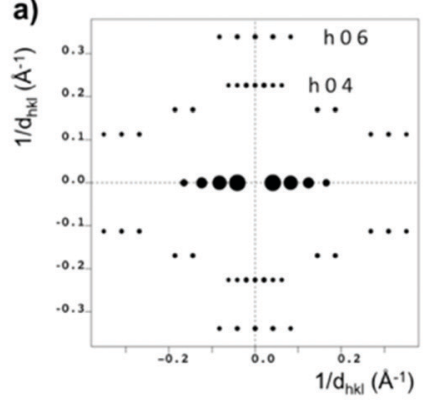

b)
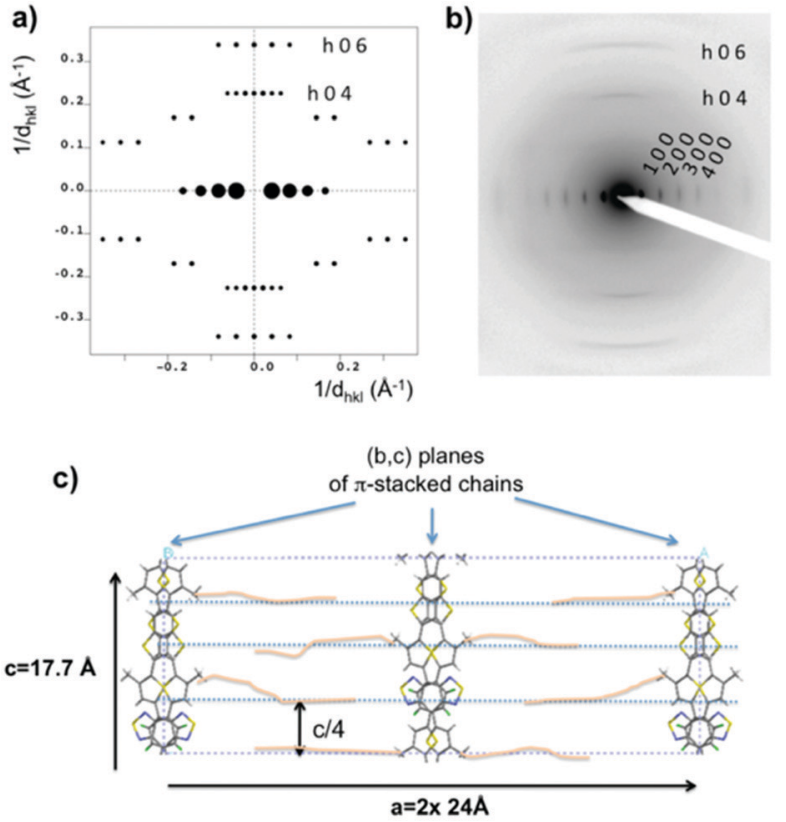

d)

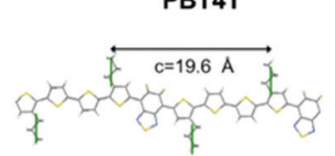

PDTBT-TT
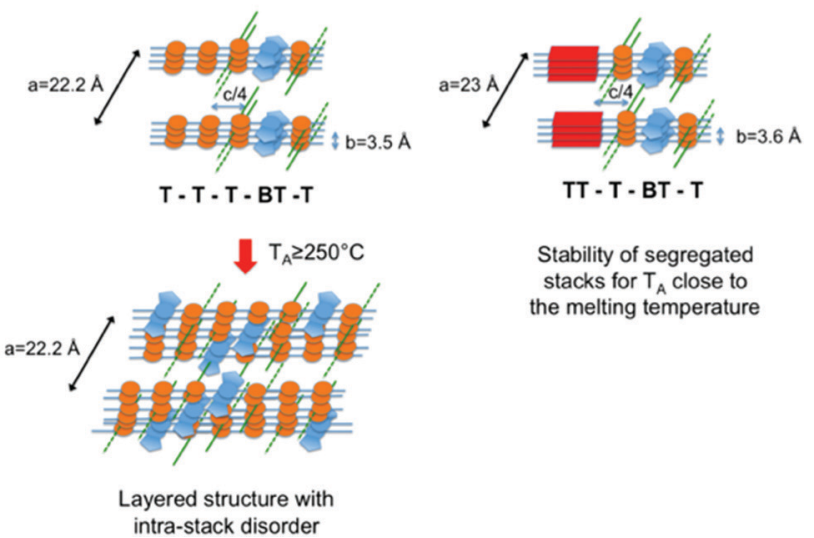

Stability of segregated stacks for $T_{A}$ close to the melting temperature

Fig. 7 Comparison of the calculated (a) and experimental (b) ED patterns for the [0 10 ] zone of PDTBT-TT. (c) (010) projection of the refined model corresponding to a segregated stacking of the chains. The unit cell is doubled along the a axis to take into account the $c / 4$ shift between successive $(b, c)$ planes of $\pi$-stacked chains. In the real structure, the shift between successive $(b, c)$ planes is given by $\pm(2 n+1) c / 4$ ( $n$ is an integer). This disorder is expected to induce a streaking of the 004 and the 006 reflections (see text) in the direction parallel to $a^{*}$ and the disappearance of the $\left(\begin{array}{lll}h & 0 & 3\end{array}\right)$ and $\left(\begin{array}{lll}h & 0 & 2\end{array}\right)$ reflections as observed in the experimental pattern in (b). (d) Segregated packing in PDTBT-TT and PBT4T. One $(b, c)$ layer over two is statistically shifted by $\pm(2 n+1) c / 4$ to allow for side chain interdigitation. Reproduced and adapted from ref. 59 (ACS).

PCPDTBT and analogues. In the following, we will focus on poly[2,6-(4,4-bis-(2-ethylhexyl)-4H-cyclopenta[2,1- $\left.b ; 3,4-b^{\prime}\right]$ dithiophene)-alt-4,7-(2,1,3-benzothiadiazole)] (PCPDTBT) and its fluorinated counterpart poly[2,6-(4,4-bis-(2-ethylhexyl)-4H-cyclopenta[2,1- $\left.b ; 3,4-b^{\prime}\right]$ dithiophene)-alt-4,7-(5-fluoro-2,1,3-benzothiadiazole)] 
(F-PCPDTBT). PCPDTBT was first introduced as a low bandgap polymer of interest for the fabrication of bulk heterojunction solar cells. $^{60}$ Optimizing the morphology in the bulk heterojunctions comprising PCPDTBT led to power conversion efficiencies (PCEs) up to $5.5 \% .^{61}$ The fluorination of the benzothiadiazole unit helped further improve PCE's up to $6.6 \%$ in blends of F-PCPDTBT with $\left[6,6^{\prime}\right]$-phenyl $\mathrm{C}_{71}$ butyric acid methyl ester $\left(\mathrm{PC}_{71} \mathrm{BM}\right){ }^{62}$ In an attempt to understand structure-property correlations, Brinkmann and Ludwigs joined their efforts to prepare highly ordered thin films for this class of polymers and analyze their structure by TEM. ${ }^{4,39,63,64}$ Interestingly, the same thermodynamically stable polymorphs (form I) of both PCPDTBT and F-PCPDTBT were obtained by different preparation methods e.g. solvent vapor annealing, melt-crystallization on PTFE substrates and high- $T$ rubbing. SVA generates flat-on lamellar crystal (see Fig. 8d and e)
Melt-crystallized on PTFE substrate

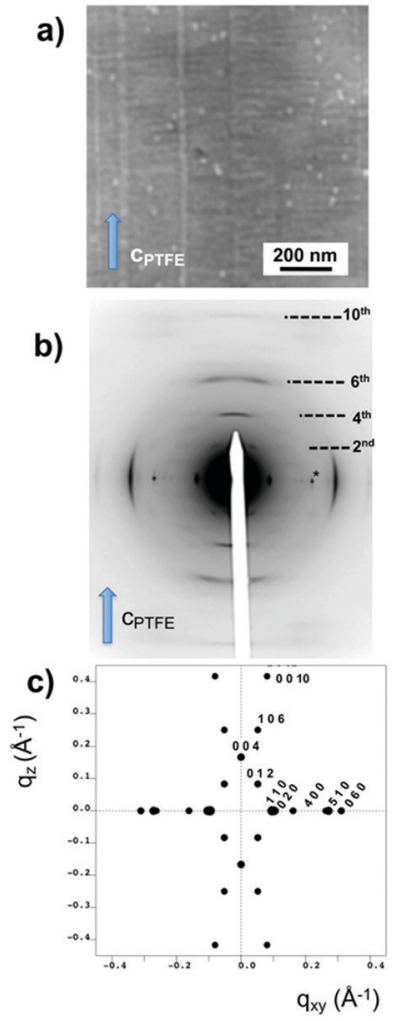

g)

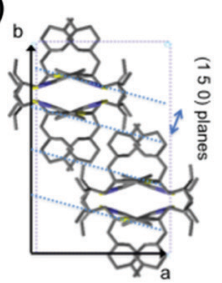

Solvent vapor annealing in chlorobenzene

d)

e)
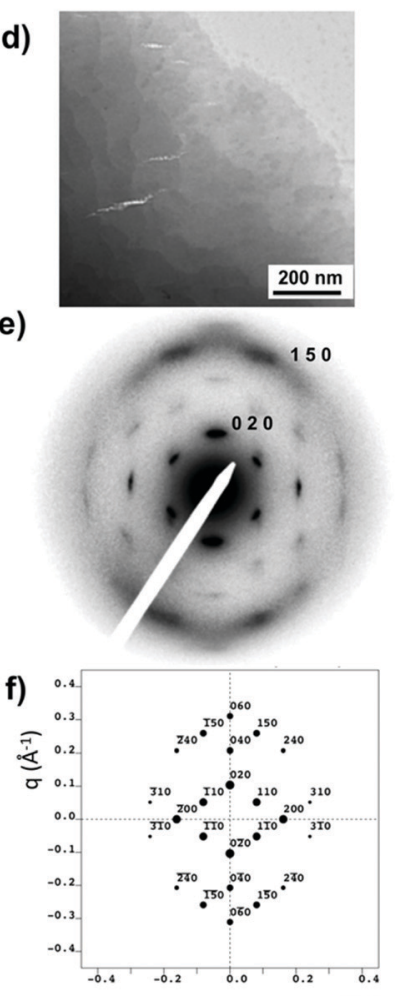

$\mathrm{q}_{z}\left(\AA^{-1}\right)$

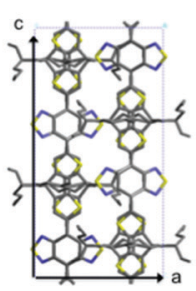

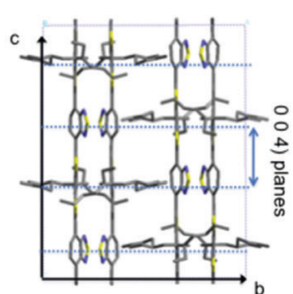

Fig. 8 Bright field images ( $a$ and $d$ ), experimental ED patterns ( $b$ and e) and calculated ED patterns ( $c$ and $f$ ) using the refined structure shown in (g) for PCPDTBT prepared by epitaxy on PTFE and solvent vapor annealing. (g) Projections of the refined structure of PCPDTBT. Reproduced with permission from ref. 64 .

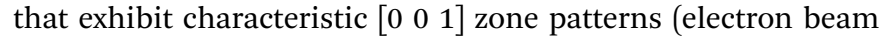
parallel to the chain direction). Alignment methods (epitaxy and rubbing) afforded highly in-plane aligned lamellae with characteristic fiber patterns for both polymers (see Fig. 8a and b). The ED patterns were analyzed by a trial-and-error method to obtain tentative structural models for both polymers. ${ }^{64}$ The refined structures are very different from the classical lamellarlike arrangement of PSCs such as P3HT and PBTTT. Indeed, the forms I of PCPDTBT and F-PCPDTBT are a high-symmetry arrangement of chains paired into dimers by $\pi$-stacking. Within the dimers, the two chains are $\pi$-stacked in a face-to-face fashion with a segregated stacking of benzothiadiazole and cyclopentadithiophene units. The side chains wrap the dimers in a similar fashion as for PFO. ${ }^{21}$ The characteristic pseudo-hexagonal diffraction pattern seen in Fig. 8d further indicates that the chain dimers organize into a pseudo-hexagonal lattice with Pccn symmetry (see Fig. 8g). The fluorination does not impact the pairing of the chains but only the way these dimers organize in the unit cell. Electron diffraction gives evidence that the lattice formed by the PCPDTBT dimers is quite disordered as indicated by the presence of broad and fuzzy reflections (especially the 15 0). Overall, the structure of form I of PCPDTBT and F-PCPDTBT is similar to that of the $\alpha$ form of poly $\left(9,9^{\prime}\right.$-dioctylfluorene) (PFO) in the sense that the structures are made by pairs of $\pi$-stacked polymer chains without long-range $\pi$-stacking. ${ }^{21}$ The two structural models of form I obtained by ED for PCPDTBT and F-PCPDTBT were further subjected to molecular dynamics simulations to probe their stability. ${ }^{39}$

All supercells built from the tentative models derived from ED data were stable and the resulting cell parameters were in very good agreement with the values determined by electron diffraction, which further validates the structural determination by ED using a trial-and-error methodology.

More generally, TEM studies on PCPDTBT-based low band gap polymers have demonstrated that this family of polymers shows an original polymorphism including amorphous, $\pi$-stacked, cross-hatched or dimer-based structures. ${ }^{39}$ The dimer-based polymorphs show the lowest charge mobilities in OFETs since charge transport is mainly along the chain direction. More interestingly, the results on the dimer structures also showed that a minor change in the polymer backbone e.g. introducing a fluorine atom on the benzothiadiazole unit can modify the packing of paired chains to a large extent. ${ }^{64}$ Both F-PCPDTBT and PCPDTBT structures involve a hexagonal-like packing of dimers of polymer chains. However, the structures differ in the orientation of the dimer in the unit cell.

This study was further extended to the case of poly[ $\left[4,4^{\prime}-\right.$ bis(2-ethylhexyl)dithiene-[3,2- $b: 2^{\prime}, 3^{\prime}$ - $\left.d\right]$ silole)-alt-4,7-(2,1,3-benzothiadiazole)] (Si-PCPDTBT) for which the bridging carbon atom of the cyclopentadithiophene block is replaced by a silicon atom. ${ }^{65}$ The polymorphism of Si-PCPDTBT differs from the PCPDTBT and F-PCPDTBT ones in that no dimer structure is formed. Instead, Si-PCPDTBT has a cross-hatched structure in solution and a classical $\pi$-stacked lamellar structure in rubbed thin films. ${ }^{66}$ This structure is stable up to very high temperatures. The different polymorphism of Si-PCPDTBT compared to the two 
analogue polymers was attributed, at least in part, to the backbone conformation and more precisely to the different angle between the cyclopentadithiophene and the benzothiadiazole units. ${ }^{39}$

PTB7. A last example of a polymer structure refined by electron diffraction is poly\{4,8-bis[(2-ethylhexyl)oxy]benzo[1,2- $b$ : 4,5- $b]$ dithiophene-2,6-diyl-alt-3-fluoro-2-[(2 ethylhexyl)carbonyl]thieno-[3,4- $b]$ thiophene-4,6-diyl $\}$ (PTB7) (see Fig. 1). PTB7 is also a high-performance PSC for solar cell applications in combination with $\mathrm{PC}_{60} \mathrm{BM}$ and $\mathrm{PCE}$ of up to $9 \%$ were reported. ${ }^{67-69}$ However, cast thin films of this polymer have a low crystallinity, which limited the structural analysis to a rough structure only. The polymer forms a lamellar structure with a $19 \AA$ interlayer stacking and a $3.9 \AA \pi$-stacking. Orientation of PTB7 was achieved

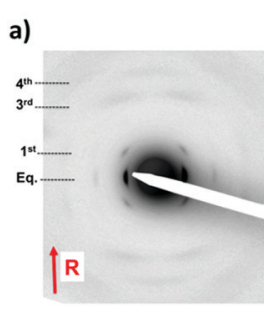

d)
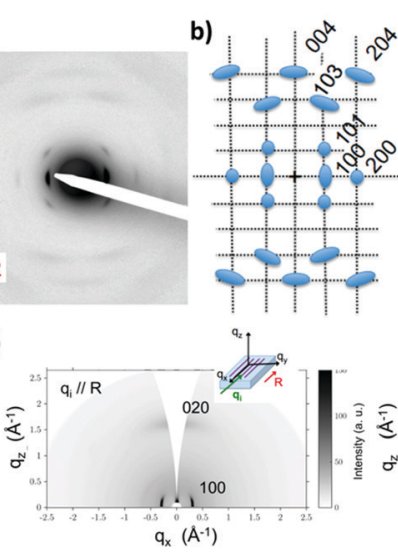

f)

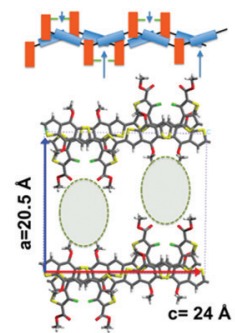

g)

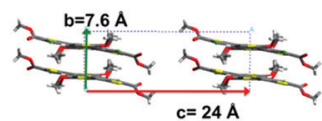

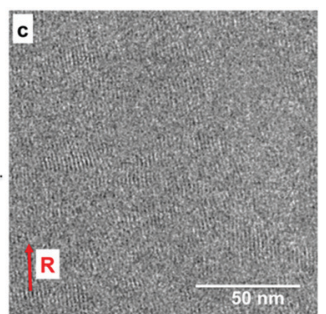

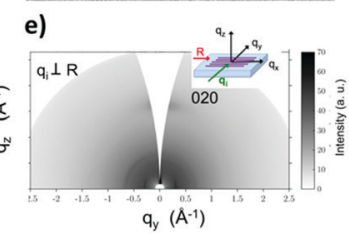

h)
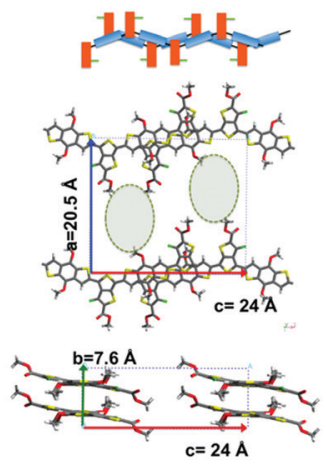

Fig. 9 (a) Experimental (a) and schematic representation (b) of the electron diffraction pattern for PTB7 films oriented by high-temperature rubbing at $200{ }^{\circ} \mathrm{C}$ followed by isothermal crystallization at $260{ }^{\circ} \mathrm{C}$ for one hour. In (a), the arrow points at the direction of rubbing (chain direction of PTB7). (c) HRTEM image showing the semi-crystalline structure of the PTB7 films. (d) and (e) GIXD patterns of the oriented PTB7 films recorded for the incident $X$-ray beam oriented parallel (d) and perpendicular (e) to the rubbing direction $R$. (f)-(i) Two possible structures labelled model 1 (f) and (g) and model 2 (h) and (i). (f) and (h) show the projections of the models 1 and 2 along the $b$-axis ( $\pi$-stacking direction). (g) and (i) show the projections of model 1 and 2 along the chain direction ( $c$ axis). For simplicity, the alkyl side chains are not shown but their location in the layered structure is highlighted by dotted ellipses. A schematic illustration of the overlap between PTB7 chains is shown. The thieno[3,4-b]thiophene and benzodithiophene groups are represented in orange and blue, respectively. The position of the fluorine atom on the thieno[3,4-b]thiophene block is illustrated in light green. In (a), the blue arrows point at the $\pi$-overlap between thiophene blocks of the benzodithiophene units in model 1 . Adapted and reproduced with permission from ref. 40 (Wiley and Sons, 2018). by high- $T$ rubbing at $200{ }^{\circ} \mathrm{C}$ followed by thermal annealing at $260{ }^{\circ} \mathrm{C}$. Such films provided oriented ED patterns as shown in Fig. 9. ${ }^{40}$ They indicate that PTB7 chains (i) adopt a layered stacking similar to e.g. P3HT (alternation of $\pi$-stacked backbones and layers of disordered alkyl side chains), (ii) show a non-planar zigzag conformation, (iii) a mixed stacking of the thieno[3,4- $b]$ thiophene and benzothiadiazole blocks imposed by the steric constraints in the packing of the branched side chains (see Fig. 9f-h). However, the low crystallinity of the PTB7 films did not allow a more accurate structural refinement. The calculation of ED patterns using a trial-and-error approach was unable to distinguish between two models shown in Fig. 9f-i. The branched alkyl side chains on the thieno[3,4- $b]$ thiophene blocks impose a steric constraint on the $\pi$-stacking of two PTB7 chains that must be shifted along the chain direction (see Fig. $9 f-h$ ). The two possible models differ by the relative shift between two $\pi$-stacked chains along the $c$ axis. PTB7 therefore illustrates the limits on structure refinement imposed by an intrinsically poor crystallization ability of the polymer. Contrary to PDOPT, PTB7 cannot crystallize readily. The branched alkyl side chains are essentially amorphous and do not form an ordered side chain sublattice as is the case for PDOPT. The crystallization abilities of side chain and backbone are highly interdependent and intimately intertwined.

\section{Conclusion}

The structure determinations of PSCs by electron diffraction illustrate the potential of this strategy when combined with controlled growth and crystallization methods. The studies, highlighted in this review, have underlined the key role of side chain interdigitation and ordering, backbone fluorination and modification of the comonomer in PSCs on their crystallization and polymorphism. Planar, twisted, zigzag or helical chain conformations were identified. Besides the well-known lamellar $\pi$-stacked morphologies, new original packing modes such as dimer structures, cross-hatched morphologies and the non $\pi$-stacked structure of PDOPT were uncovered, underlining the remarkable structural diversity of PSCs. Given the wealth of new emerging low bandgap polymers, further opportunities are at hand to improve our knowledge on structure-property correlations via a better understanding of the packing and crystallization of PSCs. In particular, for alternated copolymers, systematic studies involving a modification of one block (electron-rich or electrondeficient) keeping the other block unchanged would help draw some general rules and principles of polymer chain packing. They would serve as guidelines for the design of PSCs with enhanced optoelectronic properties. The experience gained on the PSCs studied so far will certainly be applicable to new systems such as doped-PCSs or all-conjugated block-copolymers. The structural refinement methodology may be improved by combining it with other approaches such as solid-state NMR. ${ }^{45}$

The recent infatuation for doped PSCs used for thermoelectric applications opens new and challenging opportunities for structure determination and crystallization. ${ }^{70,71}$ Materials of interest for thermoelectric applications consist of doped PSCs. 
They can be obtained by sequential doping of well crystallized PSCs with dopant molecules such as 2,3,5,6-tetrafluoro-7,7,8,8tetracyanoquinodimethane $\left(\mathrm{F}_{4}\right.$ TCNQ) ${ }^{72}$ Recent studies have demonstrated that the dopant molecules intercalate inside the crystal lattice of PSCs. ${ }^{73-75}$ The key role of dopant intercalation and ordering on the structure and thermoelectric properties of PSCs has been highlighted. Important and fundamental aspects deal with the precise position and distribution of dopant molecules in the crystalline structure of the PSCs and how it controls the electronic properties of the doped systems. On a more applied basis, crystallization and orientation of $\mathrm{TE}$ materials has emerged as a valuable and effective means to reach high TE power factors. ${ }^{74-76}$

The know-how developed in monitoring the crystallization of PSCs is essential to further optimize the structure of the doped conducting systems. New strategies for the preparation of highly ordered conducting polymers must be developed. Combining controlled growth methods of PSCs with "soft" doping methods that preserve the pristine structure of PSCs will be an interesting strategy towards highly ordered and performing conducting polymers in the form of ordered dopant/polymer intercalates. The community of plastic electronics working in the field of solar cells pays also increasing attention to all-conjugated copolymers such as P3HT- $b$-P(NDI2ODT2). They can form spontaneously nanostructured bulk heterojunctions via phase separation of donor and acceptor blocks. ${ }^{77-80}$ Preliminary studies show that the alignment methods used for the individual blocks are relevant to orient and crystallize such conjugated copolymers and ultimately study their organization by electron diffraction. In particular, TEM studies demonstrated that the crystallization of the P(NDI2ODT2) block can hamper substantially that of the P3HT block due to a mismatch of lattice parameters of the two blocks at the block junction.

\section{Conflicts of interest}

There are no conflicts to declare.

\section{Acknowledgements}

Bernard Lotz is acknowledged for careful reading of the manuscript. Financial supports from the ANR Anisotherm and CNRS grant PEPS Thermobody are gratefully acknowledged.

\section{References}

1 H. Shirakawa, E. J. Louis, A. G. MacDiarmid, C. K. Chiang and A. J. Heeger, Synthesis of electrically conducting organic polymers: halogen derivatives of polyacetylene, (CH), J. Chem. Soc., Chem. Commun., 1977, 16, 578.

2 I. F. Perepichka and D. F. Perepichka, Handbook of Thiophene-Based Materials: Applications in Organic Electronics and Photonics, John Wiley \& Sons Ltd, Chichester, UK, 2009, pp. i-xxii.
3 J. R. Reynolds, B. C. Thompson and T. A. Skotheim, Conjucated Polymers: Properties, Processing, and Applications, CRC Press, Taylor \& Francis Group, Boca Raton, FL, 2019.

4 G. L. Schulz and S. Ludwigs, Controlled Crystallization of Conjugated Polymer Films from Solution and Solvent Vapor for Polymer Electronics, Adv. Funct. Mater., 2017, 27, 1603083.

5 E. J. W. Crossland, K. Tremel, F. Fischer, K. Rahimi, G. Reiter, U. Steiner and S. Ludwigs, Anisotropic Charge Transport in Spherulitic Poly(3-hexylthiophene) Films, $A d v$. Mater., 2012, 24, 839.

6 K. Rahimi, I. Botiz, N. Stingelin, N. Kayunkid, M. Sommer, F. P. V. Koch, H. Nguyen, O. Coulembier, P. Dubois, M. Brinkmann and G. Reiter, Controllable Processes for Generating Large Single Crystals of Poly(3-hexylthiophene), Angew. Chem., Int. Ed., 2012, 51, 11131.

7 M. Brinkmann, L. Hartmann, L. Biniek, K. Tremel and $N$. Kayunkid, Orienting Semi-Conducting $\pi$-Conjugated Polymers, Macromol. Rapid Commun., 2014, 35, 9.

8 M. Brinkmann, in Conjugated polymers and oligomers: structural and soft matter aspects, ed. M. Knaapila, World Scientific, 2018, pp. 29-66.

9 J. A. Lim, F. Liu, S. Ferdous, M. Muthukumar and A. L. Briseno, Polymer Semiconductor Crystals, Mater. Today, 2010, 13, 14.

10 P. L. Boudreault, A. Najari and M. Leclerc, Processable LowBandgap Polymers for Photovoltaic Applications, Chem. Mater., 2011, 23, 456.

$11 \mathrm{~T}$. Xu and L. Yu, How to design low bandgap polymers for highly efficient organic solar cells, Mater. Today, 2014, 17, 11-15.

12 X. Zhang, L. J. Richter, D. M. DeLongchamp, R. J. Kline, M. R. Hammond, I. McCulloch, M. Heeney, R. S. Ashraf, J. N. Smith and T. D. Anthopoulos, Molecular Packing of High-Mobility Diketo Pyrrolo-Pyrrole Polymer Semiconductors with Branched Alkyl Side Chains, J. Am. Chem. Soc., 2011, 133, 15073.

13 Z. Chen, Y. Zheng, H. Yan and A. Facchetti, Naphthalenedicarboximide- vs. Perylenedicarboximide-Based Copolymers. Synthesis and Semiconducting Properties in Bottom-Gate N-Channel Organic Transistors, J. Am. Chem. Soc., 2009, 131, 8-9.

14 H. Zhou, L. Yang, A. C. Stuart, S. C. Price, S. Liu and W. You, Donor-Acceptor Polymers Incorporating Alkylated Dithienylbenzothiadiazole for Bulk Heterojunction Solar Cells: Pronounced Effect of Positioning Alkyl Chains, Macromolecules, 2011, 50, 2995.

15 M. Zhang, X. Guo, S. Zhang and J. Hou, Synergistic Effect of Fluorination on Molecular Energy Level Modulation in Highly Efficient Photovoltaic Polymers, Adv. Mater., 2013, 26, 1118.

16 N. Leclerc, P. Chavez, O. A. Ibraikulov, T. Heiser and P. Lévêque, Impact of Backbone Fluorination on $\pi$-Conjugated Polymers in Organic Photovoltaic Devices, Polymers, 2016, 8, 11.

17 Y. Li, P. Sonar, L. Murphy and W. Hong, High mobility diketopyrrolopyrrole (DPP)-based organic semiconductor materials for organic thin film transistors and photovoltaics, Energy Environ. Sci., 2013, 6, 1684. 
18 M. Sommer, Conjugated polymers based on naphthalene diimide for organic electronics, J. Mater. Chem. C, 2014, 2, 3088.

19 S. Ludwigs, P3HT revisited - From Molecular Scale to Devices, Springer, Berlin, Heidelberg, 2014.

20 M. Brinkmann and P. Rannou, Molecular Weight Dependence of Chain Packing and Semicrystalline Structure in Oriented Films of Regioregular Poly(3-hexylthiophene) Revealed by High-Resolution Transmission Electron Microscopy, Macromolecules, 2009, 42, 1125.

21 M. Brinkmann, Directional Epitaxial Crystallization and Tentative Crystal Structure of Poly(9,9'-di-n-octyl-2,7-fluorene), Macromolecules, 2007, 40, 7532.

22 E. Mena-Osteriz, Superstructures of Self-Organizing Thiophenes, Adv. Mater., 2002, 14, 609.

23 M. Brinkmann, Structure and morphology control in thin films of regioregular poly(3-hexylthiophene), J. Polym. Sci., Part B: Polym. Phys., 2011, 49, 1218.

24 D. J. Blundell, A. Keller and A. J. Kovacs, A new selfnucleation phenomenon and its application to the growing of polymer crystals from solution, J. Polym. Sci., Part B: Polym. Phys., 1966, 4, 481.

25 J. C. Wittmann, A. M. Hodge and B. Lotz, Epitaxial crystallization of polymers onto benzoic acid: polyethylene and paraffins, aliphatic polyesters, and polyamides, J. Polym. Sci., Part B: Polym. Phys., 1983, 21, 2495.

26 M. Brinkmann and J.-C. Wittmann, Orientation of Regioregular Poly(3-hexylthiophene) by Directional Solidification: A Simple Method to Reveal the Semicrystalline Structure of a Conjugated Polymer, Adv. Mater., 2006, 18, 860.

27 M. Brinkmann, C. Contal, N. Kayunkid, T. Djuric and R. Resel, Highly Oriented and Nanotextured Films of Regioregular Poly(3-hexylthiophene) Grown by Epitaxy on the Nanostructured Surface of an Aromatic Substrate, Macromolecules, 2010, 43, 7604.

28 A. Hamidi-Sakr, D. Schiefer, S. Covindarassou, L. Biniek, M. Sommer and M. Brinkmann, Macromolecules, 2016, 49, 3452.

29 M. Brinkmann, N. Charoenthai, R. Traiphol, P. Piyakulawat, J. Wlosnewski and U. Asawapirom, Highly Oriented and Crystalline Films of a Phenyl-Substituted Polythiophene Prepared by Epitaxy: Structural Model and Influence of Molecular Weight, Macromolecules, 2009, 42, 8298.

30 H. Zhou, S. Jiang and S. Yan, Epitaxial Crystallization of Poly(3-hexylthiophene) on a Highly Oriented Polyethylene Thin Film from Solution, J. Phys. Chem. B, 2011, 15, 13449.

31 W. A. Memon, J. Li, Q. Fang, Z. Ren, S. Yan and X. Sun, Synergistic Effect of Solvent and Epitaxy on the Formation of Anisotropic Structures of P3HT and P3HT/PCBM Films, J. Phys. Chem. B, 2019, 123, 7233-7239.

32 M. Brinkmann, F. Chandezon, C. Julien-Raban and R. P. Pansu, Epitaxial Growth of Highly Oriented Fibers of Semiconducting Polymers with a Shish-Kebab-Like Superstructure, Adv. Funct. Mater., 2009, 19, 2759.

33 B. O’Connor, R. J. Kline, B. R. Conrad, L. J. Richter, D. Gundlach, M. F. Toney and D. M. DeLongchamp, Anisotropic Structure and Charge Transport in Highly Strain-Aligned Regioregular Poly(3hexylthiophene), Adv. Funct. Mater., 2011, 21, 3697.
34 S. Nagamatsu, W. Takashima, K. Kaneto, Y. Yoshida, N. Tanigaki, K. Yase and K. Omote, Backbone Arrangement in "Friction-Transferred" Regioregular Poly(3-alkylthiophene)s, Macromolecules, 2003, 36, 5252.

35 L. Biniek, N. Leclerc, T. Heiser, R. Bechara and M. Brinkmann, Large Scale Alignment and Charge Transport Anisotropy of pBTTT Films Oriented by High Temperature Rubbing, Macromolecules, 2013, 46, 4014-4023.

36 L. Biniek, S. Pouget, D. Djurado, E. Gonthier, K. Tremel, N. Kayunkid, E. Zaborova, N. Crespo-Monteiro, O. Boyron, N. Leclerc, S. Ludwigs and M. Brinkmann, High-Temperature Rubbing: A Versatile Method to Align $\pi$-Conjugated Polymers without Alignment Substrate, Macromolecules, 2014, 47, 3871.

37 A. Hamidi-Sakr, L. Biniek, S. Fall and M. Brinkmann, Precise Control of Lamellar Thickness in Highly Oriented Regioregular Poly(3-Hexylthiophene) Thin Films Prepared by HighTemperature Rubbing: Correlations with Optical Properties and Charge Transport, Adv. Funct. Mater., 2016, 26, 408.

38 N. Kayunkid, S. Uttiya and M. Brinkmann, Structural Model of Regioregular Poly(3-hexylthiophene) Obtained by Electron Diffraction Analysis, Macromolecules, 2010, 43, 4961.

39 G. L. Schulz, F. S. U. Fischer, D. Trefz, A. Melnyk, A. HamidiSakr, M. Brinkmann, D. Andrienko and S. Ludwigs, The PCPDTBT Family: Correlations between Chemical Structure, Polymorphism, and Device Performance, Macromolecules, 2017, 50, 1402.

40 L. Biniek, A. Hamidi-Sakr, L. Grodd, S. Escoubas, Y. J. Dappe, S. Grigorian, M. Schmutz and M. Brinkmann, Structure and Charge Transport Anisotropy of Polythieno[3,4-b]Thiophene-co-Benzodithiophene (PTB7) Oriented by HighTemperature Rubbing, Adv. Electron. Mater., 2018, 4, 1700480.

41 T. J. Prosa, M. J. Winokur and R. D. McCullough, Evidence of a Novel Side Chain Structure in Regioregular Poly(3alkylthiophenes), Macromolecules, 1996, 29, 3654.

42 K. Tashiro, M. Kobayashi, T. Kawai and K. Yoshino, Crystal structural change in poly(3-alkyl thiophene)s induced by iodine doping as studied by an organized combination of X-ray diffraction, infrared/Raman spectroscopy and computer simulation techniques, Polymer, 1997, 38, 2867.

43 C. Poelking, K. Daoulas, A. Troisi and D. Andrienko, Morphology and Charge Transport in P3HT: A Theorist's Perspective, Adv. Polym. Sci., 2014, 265, 139.

44 S. Dag and L. W. Wang, Packing Structure of Poly(3hexylthiophene) Crystal: Ab Initio and Molecular Dynamics Studies, J. Phys. Chem. B, 2010, 114, 5997.

45 D. Dudenko, A. Kiersnowski, J. Shu, W. Pisula, D. Sebastiani, H. W. Spiess and M. R. Hansen, A Strategy for Revealing the Packing in Semicrystalline $\pi$-Conjugated Polymers: Crystal Structure of Bulk Poly-3-hexyl-thiophene (P3HT), Angew. Chem., Int. Ed., 2012, 51, 11068.

46 A. Zhugayevych, O. Mazaleva, A. Naumov and S. Tretiak, Lowest-Energy Crystalline Polymorphs of P3HT, J. Phys. Chem. C, 2018, 122, 9141-9151.

47 Y. Wang, B. Heck, D. Shiefer, J. O. Agumba, M. Sommer, T. Wen and G. Reiter, Anisotropic Photophysical Properties 
of Highly Aligned Crystalline Structures of a Bulky Substituted Poly(thiophene), ACS Macro Lett., 2014, 3, 881.

48 D. Schiefer, T. Wen, Y. Wang, P. Goursot, H. Komber, R. Hanselmann, P. Braunstein, G. Reiter and M. Sommer, Nickel Catalyst with a Hybrid P, N Ligand for Kumada Catalyst Transfer Polycondensation of Sterically Hindered Thiophenes, ACS Macro Lett., 2014, 3, 617.

49 A. M. Buono, N. H. Son, G. Raos, L. Gila, A. Cominetti, M. Castellani and S. V. Meille, Macromolecules, 2010, 43, 6772 .

50 K. E. Aasmundveit, E. J. Samuelsen, W. Mammo, M. Svensson, M. R. Anderson, L. A. A. Peterson and O. Inganäs, Macromolecules, 2000, 33, 5481.

51 J. T. E. Quinn, J. Zhu, X. Li, J. Wang and Y. Li, Recent progress in the Development of n-type Organic Semiconductors for Organic Field Effect Transistors, J. Mater. Chem. C, 2017, 5, 8654.

52 H. Yan, Z. Chen, C. Newman, J. R. Quinn, F. Dötz, M. Kastler and A. Facchetti, A High-Mobility Electron-Transporting Polymer For Printed Transistors, Nature, 2009, 457, 679.

53 M. Brinkmann, E. Gonthier, S. Bogen, K. Tremel, S. Ludwigs, M. Hufnagel and M. Sommer, Segregated versus Mixed Interchain Stacking in Highly Oriented Films of Naphthalene Diimide Bithiophene Copolymers, ACS Nano, 2012, 6, 10319.

54 K. Tremel, F. S. U. Fischer, N. Kayunkid, R. Di Pietro, R. Tkachov, A. Kiriy, D. Neher, S. Ludwigs and M. Brinkmann, Charge Transport Anisotropy in Highly Oriented Thin Films of the Acceptor Polymer P(NDI2OD-T2), Adv. Energy Mater., 2014, 4, 1301659.

55 D. Trefz, Y. M. Gross, C. Dingler, R. Tkachov, A. Hamidi Sakr, A. Kiriy, C. R. McNeill, M. Brinkmann and S. Ludwigs, Tuning Orientational Order of Highly Aggregating P(NDI2OD-T2) by Solvent Vapor Annealing and Blade Coating, Macromolecules, 2019, 52, 43.

56 O. A. Ibraikulov, C. Ngov, P. Chàvez, I. Bulut, B. Heinrich, O. Boyron, K. L. Gerasimov, D. A. Ivanov, S. Swaraj, S. Méry, N. Leclerc, P. Lévêque and T. Heiser, Face-on orientation of fluorinated polymers conveyed by long alkyl chains: a prerequisite for high photovoltaic performances, J. Mater. Chem. A, 2018, 6, 12038.

57 S. Zhang, B. Yang, D. Liu, H. Zhang, W. Zhao, Q. Wang, C. He and J. Hou, Correlations among Chemical Structure, Backbone Conformation, and Morphology in Two Highly Efficient Photovoltaic Polymer Materials, Macromolecules, 2016, 49, 120-126.

58 A. Efrem, K. Wang, T. Jia and M. Wang, Direct arylation polymerization toward a narrow bandgap donor-acceptor conjugated polymer of alternating 5,6-difluoro-2,1,3-benzothiadiazole and alkyl-quarternarythiophene: from synthesis, optoelectronic properties to devices, J. Polym. Sci., Part A: Polym. Chem., 2017, 55, 1869-1879.

59 Y. Zhong, L. Biniek, N. Leclerc, S. Ferry and M. Brinkmann, Segregated versus Disordered Stacking in Two Low Bandgap Alternated Copolymers for Photovoltaic Applications: Impact of Polymorphism on Optical Properties, Macromolecules, 2018, 51, 4238.
60 M. C. Scharber, D. Mühlbacher, M. Koppe, P. Denk, C. Waldauf, A. J. Heeger and C. J. Brabec, Design Rules for Donors in Bulk-Heterojunction Solar Cells-Towards 10\% Energy-Conversion Efficiency, Adv. Mater., 2006, 18, 789.

61 J. Peet, J. Y. Kim, N. E. Coates, W. L. Ma, D. Moses, A. J. Heeger and G. C. Bazan, Efficiency enhancement in lowbandgap polymer solar cells by processing with alkane dithiols, Nat. Mater., 2007, 6, 497.

62 S. Albrecht, S. Janietz, W. Schindler, J. Frisch, J. Kurpiers, J. Kniepert, S. Inal, P. Pingel, K. Fostiropoulos, N. Koch and D. Neher, Fluorinated Copolymer PCPDTBT with Enhanced Open-Circuit Voltage and Reduced Recombination for Highly Efficient Polymer Solar Cells, J. Am. Chem. Soc., 2012, 134, 14932.

63 F. S. U. Fischer, D. Trefz, J. Back, N. Kayunkid, B. Tornow, S. Albrecht, K. Yager, G. Singh, A. Karim, D. Neher, M. Brinkmann and S. Ludwigs, Highly Crystalline Films of PCPDTBT with Branched Side Chains by Solvent Vapor Crystallization: Influence on Opto-Electronic Properties, Adv. Mater., 2015, 27, 1223.

64 F. S. U. Fischer, N. Kayunkid, D. Trefz, S. Ludwigs and M. Brinkmann, Structural Models of Poly(cyclopentadithiophene-alt-benzothiadiazole) with Branched Side Chains: Impact of a Single Fluorine Atom on the Crystal Structure and Polymorphism of a Conjugated Polymer, Macromolecules, 2015, 48, 3974.

65 J. Hou, H.-Y. Chen, S. Zhang, G. Li and Y. Yang, Synthesis, Characterization, and Photovoltaic Properties of a Low Band Gap Polymer Based on Silole-Containing Polythiophenes and 2,1,3-Benzothiadiazole, J. Am. Chem. Soc., 2008, 130, 16144.

66 C. J. Takacs, M. A. Brady, N. D. Treat, E. J. Kramer and M. L. Chabinyc, Quadrites and Crossed-Chain Crystal Structures in Polymer Semiconductors, Nano Lett., 2014, 14, 3096.

67 S. B. Dkhil, M. Pfannmöller, S. Bals, T. Koganezawa, N. Yoshimoto, D. Hannani, M. Gaceur, C. Videlot-Ackermann, O. Margeat and J. Ackermann, Square-Centimeter-Sized HighEfficiency Polymer Solar Cells: How the Processing Atmosphere and Film Quality Influence Performance at Large Scale, Adv. Energy Mater., 2016, 6, 1600290.

68 S. Park, J. Jeong, G. Hyun, M. Kim, H. Lee and Y. Yi, The origin of high PCE in PTB7 based photovoltaics: proper charge neutrality level and free energy of charge separation at PTB7/PC71BM interface, Sci. Rep., 2016, 6, 35262.

69 L. Lu and L. Yu, Thermoelectric plastics: from design to synthesis, processing and structure-property relationships, Adv. Mater., 2014, 26, 4413.

70 R. Kroon, D. A. Mengistie, D. Kiefer, J. Hynynen, J. D. Ryan, L. Yu and C. Müller, Thermoelectric plastics: from design to synthesis, processing and structure-property relationships, Chem. Soc. Rev., 2016, 45, 6147.

71 J. Hynynen, D. Kiefer, L. Yu, R. Kroon, R. Munir, A. Amassian, M. Kemerink and C. Müller, Enhanced Electrical Conductivity of Molecularly p-Doped Poly(3-hexylthiophene) through Understanding the Correlation with Solid-State Order, Macromolecules, 2017, 50, 8140.

72 D. T. Scholes, P. Y. Yee, J. R. Lindemuth, H. Kang, J. Onorato, R. Ghosh, C. K. Luscombe, F. C. Spano, S. H. Tolbert and 
B. J. Schwartz, The Effects of Crystallinity on Charge Transport and the Structure of Sequentially Processed F4TCNQDoped Conjugated Polymer Films, Adv. Funct. Mater., 2017, 27, 1702654.

73 A. Hamidi-Sakr, L. Biniek, J.-L. Bantignies, D. Maurin, L. Herrmann, N. Leclerc, P. Lévêque, V. Vijayakumar, N. Zimmermann and M. Brinkmann, A Versatile Method to Fabricate Highly In-Plane Aligned Conducting Polymer Films with Anisotropic Charge Transport and Thermoelectric Properties: The Key Role of Alkyl Side Chain Layers on the Doping Mechanism, Adv. Funct. Mater., 2017, 27, 1700173.

74 V. Vijayakumar, Y. Zhong, V. Untilova, M. Bahri, L. Herrmann, L. Biniek, N. Leclerc and M. Brinkmann, Bringing Conducting Polymers to High Order: Toward Conductivities beyond $10^{5} \mathrm{~S} \mathrm{~cm}^{-1}$ and Thermoelectric Power Factors of $2 \mathrm{~mW} \mathrm{~m}^{-1} \mathrm{~K}^{-2}$, Adv. Energy Mater., 2019, 9, 1900266.

75 V. Vijayakumar, E. Zaborova, L. Biniek, H. Zeng, L. Herrmann, A. Carvalho, O. Boyron, N. Leclerc and M. Brinkmann, Effect of Alkyl Side Chain Length on Doping Kinetics, Thermopower, and Charge Transport Properties in Highly Oriented F4TCNQ-Doped PBTTT Films, ACS Appl. Mater. Interfaces, 2019, 11, 4942-4953.
76 J. Wang, Y. Liu, H. Li, S. Yan, X. Sun, D. Tu, X. Guo and Z. Ren, Enhanced charge transport and thermoelectric performance of P(NDI2OD-T2) by epitaxial crystallization on highly oriented polyethylene substrates, Mater. Chem. Front., 2020, 4, 661-668.

77 V. Untilova, T. Biskup, L. Biniek, V. Vijayakumar and M. Brinkmann, Control of Chain Alignment and Crystallization Helps Enhance Charge Conductivities and Thermoelectric Power Factors in Sequentially Doped P3HT:F4TCNQ Films, Macromolecules, 2020, 53, 2441-2453.

78 F. Nübling, D. Yang, P. Müller-Buschbaum, M. Brinkmann and M. Sommer, In Situ Synthesis of Ternary Block Copolymer/ Homopolymer Blends for Organic Photovoltaics, ACS Appl. Mater. Interfaces, 2018, 10, 18149-18160.

79 F. Nübling, T. R. Hopper, B. Kuei, H. Komber, V. Untilova, S. B. Schmidt, M. Brinkmann, E. D. Gomez, A. A. Bakulin and M. Sommer, Block Junction-Functionalized All-Conjugated Donor-Acceptor Block Copolymers, ACS Appl. Mater. Interfaces, 2019, 11, 1143-1155.

80 V. Untilova, F. Nübling, L. Biniek, M. Sommer and M. Brinkmann, Impact of Competing Crystallization Processes on the Structure of All-Conjugated Donor-Acceptor Block Copolymers P3HT- $b$-PNDIT2 in Highly Oriented Thin Films, ACS Appl. Polym. Mater., 2019, 1, 1660. 